Chirurg 2011 · 82:293-294

DOI 10.1007/s00104-011-2085-9

Online publiziert: 18. März 2011

(c) Springer-Verlag 2011

\author{
A. Haverich \\ Klinik für Herz-, Thorax-, Transplantations- und Gefäßchirurgie, \\ Medizinische Hochschule Hannover
}

\title{
Tissue Engineering in der Herzchirurgie
}

les Gewebe herzustellen. Das kardiovaskuläre Tissue Engineering spielt in diesem vielversprechenden Bereich der medizinischen Forschung eine führende Rolle, nicht zuletzt, weil die meisten der heutigen kardiovaskulären, chirurgischen Behandlungen einen Ersatz von erkrankten Geweben und Organen vorsehen. Dabei könnte keines der bisher für den Ersatz kardiovaskulärer Strukturen erhältlichen Materialien als ideale Prothese beschrieben werden. Mechanische und biologische Herzklappenprothesen unterliegen nach wie vor den bekannten Einschränkungen, wie der Notwendigkeit der lebenslangen Antikoagulation oder der frühen Degeneration. Allerdings gibt es Hinweise aus der jüngeren Vergangenheit, dass implantierte, dezellularisierte Homografts in Pulmonalposition bei Kindern zum Wachstum und damit zur Regeneration befähigt sind. Erste Erfolge nach Implantation in Aortenposition versprechen sogar eine noch breitere Anwendbarkeit dieses Konzepts.

\section{(7) Erfolge bei der Vaskularisierung myokardialen Ersatzgewebes eröffnen neue Perspektiven}

Die Herztransplantation als bisheriger Goldstandard der chirurgischen Therapie der schweren Herzinsuffizienz wird auch weiterhin durch ungenügende Spenderorgane einer fatalen Limitation unterliegen. Die Rekonstruktion geschädigten Myokards mittels regenerativer Ersatzma- terialien könnte hier Abhilfe schaffen. Ein wesentliches Hindernis bei der Erzeugung myokardialen Ersatzgewebes von ausreichender Stärke, das den hohen mechanischen Anforderungen im Hochdruckbereich des Herzens standhält, stellt die Versorgung mit Sauerstoff und Nährstoffen dar. Die jüngsten Erfolge bei der Vaskularisierung eröffnen auch in diesem Feld neue Perspektiven.

Schließlich gibt es Ansätze zur Züchtung von kleinlumigen Gefäßprothesen, mit denen der hohen Verschlussrate aufgrund von thrombotischen Ablagerungen oder Intimahyperplasien begegnet wird. Hierbei werden natürliche Substanzen, wie z. B. Kollagen und Fibrin, sowie synthetische Materialien, wie z. B. Polymilchsäure und Polycaprolacton oder Hybride, bisher vorwiegend in vitro oder in Tiermodellen getestet.

In allen Segmenten des kardiovaskulären Tissue Engineerings werden zellfreie synthetische und künstliche Substrate untersucht, die entweder auf soliden vorbestehenden Gerüsten basieren oder über eine De-novo-Synthese erzeugt werden. Über die Repopulation dieser zellfreien Strukturen mit embryonalen Stammzellen oder (induzierten) pluripotenten Stammzellen in geeigneten Bioreaktorsystemen oder auch erst im Körper des Empfängers, scheint die Funktionalisierung aller bioartifizieller Ersatzgewebe zur Therapie geschädigter kardiovaskulärer Strukturen möglich zu werden. In naher Zukunft werden unsere Pa- 
tienten, die bisher mit nichtidealen Implantaten oder gar nicht versorgt werden konnten, von dieser Technologie profitieren. Ökonomische und rechtliche Aspekte sowie technische und ethische Fragen sind heute noch nicht abschließend geklärt. Das kardiovaskuläre Tissue Engineering hat jedoch einen fortgeschrittenen Stand der Entwicklung erreicht, welcher den Lesern dieser Ausgabe von Der Chirurg für Herzklappen, Myokard, Gefäße und der Auswahl geeigneter Zellquellen vorgestellt wird.

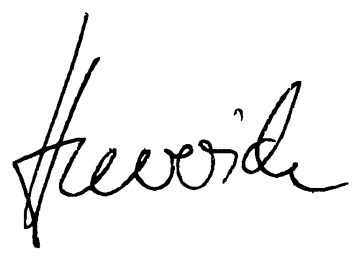

Prof. Dr. Axel Haverich

\section{Korrespondenzadresse}

\section{Prof. Dr. A. Haverich}

Klinik für Herz-, Thorax-, Transplantationsund Gefäßchirurgie,

Medizinische Hochschule Hannover, Carl-Neuberg-Straße 1, 30625 Hannover haverich.axel@mh-hannover.de

\section{Ausschreibung}

\section{Oskar und Helene-Medizin-Preis 2011}

\section{Stiftung Oskar Helene Heim}

Die Stiftung Oskar-Helene-Heim, deren Zweck die Förderung der Wissenschaft und Forschung ist, verleiht seit 2010 jährlich den mit $50.000 €$ dotierten Oskar und Helene-Medizin-Preis. Die Schirmherrschaft für diesen Preis hat die Bundesministerin für Bildung und Forschung, Frau Prof. Dr. Annette Schavan, übernommen. Mit diesem Medizinpreis sollen hervorragende Leistungen, insbesondere auf einem der folgenden Gebiete gewürdigt werden: Orthopädie und Orthopädie-Technik, Pneumologie sowie Gastroenterologie/Viszeralchirurgie.

Der Preis wird im Jahr 2011

auf dem Gebiet der

Orthopädie und Unfallchirurgie

ausgeschrieben. Die Behandlung von nichtheilenden Frakturen stellt eine schwerwiegende Problematik dar und führt zu keinem befriedigenden Ergebnis. Im Bereich der Endoprothetik ist insbesondere nach Lockerungssituationen eine neuerliche Verankerung von Implantaten nicht möglich, da der Knochen seine Wachstumspotenz eingebüßt hat. Mit dem Preis soll daher ein habilitierter Mediziner ausgezeichnet werden, der bei der

"Stimulation der Knochenheilung bei Frakturen und / oder der Osteointegration von Endoprothesen"

besonders relevante Ergebnisse der Grundlagen- und/oder klinischen Forschung in Deutschland erzielt hat. Der prämierte Erkenntnisgewinn soll einer breiten Öffentlichkeit vermittelbar sein. Dem Antrag sollen der Lebenslauf, die fünf wichtigsten Arbeiten aus den letzten drei Jahren zum Thema „Stimulation der Knochenheilung bei Frakturen und/ oder der Osteointegration von Endopro- thesen" sowie eine inhaltliche Zusammenfassung der Forschungsergebnisse beigefügt werden.

Das Preisgeld ist für Forschungszwecke nach der freien Entscheidung des Preisträgers zu verwenden. Der Preisträger hat der Stiftung diese Verwendung in geeigneter Weise zu belegen.

Der Antrag ist bis zum 30. Juni 2011

bei der Stiftung

Oskar-Helene-Heim einzureichen

(www.stiftung-ohh.de).

Weitere Informationen erteilt der Geschäftsführer der Stiftung

\section{Werner Ukas}

werner.ukas@helios-kliniken.de oder info@stiftung-ohh.de

Tel. 030/8102-1100

Die Preisverleihung wird im Oktober 2011 in Berlin erfolgen.

Zweck der Stiftung Oskar-Helene-Heim ist die Förderung von Wissenschaft und Forschung in der Medizin, insbesondere der Orthopädie, der Lungenheilkunde, Gastroenterologie und Viszeralchirurgie sowie der Orthopädietechnik. Zu diesem Zweck unterstützt die Stiftung Forschungsprojekte oder sonstige gemeinnützige gesundheitsfördernde Vorhaben, verleiht Stipendien und vergibt jährlich den Oskar und Helene-Medizinpreis. Die Stiftung ist darüber hinaus Teilhaber am HELIOS Klinikum Emil von Behring im Berliner Südwesten. Seit 2004 besteht mit der HELIOS Kliniken GmbH durch die Eingliederung der damaligen Zentralklinik Emil von Behring eine strategische Partnerschaft. Gegründet wurde die Stiftung 1966. Bis zum Jahr 2000 war sie Träger des renommierten orthopädischen Krankenhauses Oskar-HeleneHeim, von 2001 bis 2004 der Zentralklinik Emil von Behring. Die Wurzeln der Stiftung reichen zurück ins Jahr 1905, als der Industrielle Oskar Pinsch gemeinsam mit seiner Ehefrau Helene einen Verein gründete, dessen Ziel die Heilung, Ausbildung und Überwachung junger Menschen mit gesundheitlichem Handicap war. 